\title{
ARTICLE
}

\section{Identification of the Finnish founder mutation for diastrophic dysplasia (DTD)}

\author{
Johanna Hästbacka ${ }^{1}$, Anne Kerrebrock ${ }^{2}$, Kati Mokkala ${ }^{1}$, Gregory Clines ${ }^{3}$, \\ Michael Lovett ${ }^{3,4}$, Ilkka Kaitila ${ }^{5}$, Albert de la Chapelle ${ }^{6}$ and Eric S Lander ${ }^{2,7}$ \\ ${ }^{1}$ Department of Medical Genetics, Haartman Institute, University of Helsinki, Finland \\ ${ }^{2}$ Whitehead Institute for Biomedical Research, Cambridge, Massachusetts, USA \\ ${ }^{3}$ McDermott Center and ${ }^{4}$ Department of Biochemistry, University of Texas Southwestern Medical Center at Dallas, \\ Texas, USA \\ ${ }^{5}$ Department of Clinical Genetics, Helsinki University Central Hospital, Finland \\ ${ }^{6}$ Division of Human Cancer Genetics, Ohio State University, Columbus, Ohio, USA \\ ${ }^{7}$ Department of Biology, Massachusetts Institute of Technology, Cambridge, Massachusetts, USA
}

\begin{abstract}
Diastrophic dysplasia (DTD) is especially prevalent in Finland and the existence of a founder mutation has been previously inferred from the fact that $95 \%$ of Finnish DTD chromosomes have a rare ancestral haplotype found in only $4 \%$ of Finnish control chromosomes. Here we report the identification of the Finnish founder mutation as a GT- $>$ GC transition $(c .-26+2 T>C)$ in the splice donor site of a previously undescribed 5'-untranslated exon of the diastrophic dysplasia sulfate transporter gene (DTDST); the mutation acts by severely reducing mRNA levels. Among 84 DTD families in Finland, patients carried two copies of the mutation in 69 families, one copy in 14 families, and no copies in one family. Roughly $90 \%$ of Finnish DTD chromosomes thus carry the splice-site mutation, which we have designated DTDST $_{\text {Fin }}$. Unexpectedly, we found that nine of the DTD chromosomes having the apparently ancestral haplotype did not carry DTDST $_{\text {Fin }}$, but rather two other mutations. Eight such chromosomes had an R279W mutation and one had a V340del deletion. We consider the possible implications of presence of multiple DTD mutations on this rare haplotype.
\end{abstract}

Keywords: splice site mutation; DTDST; disease mutation; skeletal dysplasia

\section{Introduction}

Diastrophic dysplasia (DTD, MIM No. 222600) is a recessive chondrodysplasia whose main clinical features are short-limbed dwarfism and generalized dysplasia of the joints. Other disease characteristics variably include spinal deformity, peculiar club-foot deformity, cleft palate, deformed ear pinnae, displaced

Correspondence: Johanna Hästbacka, Department of Medical Genetics, Haartman Institute, University of Helsinki, 00014 Helsinki, Finland. Tel: + 3589 19126791; E-mail: johanna.hastbacka@helsinki.fi

Received 11 March 1999; accepted 10 May 1999 and subluxated thumbs (often hitch-hiker thumbs), and flexion limitations of the proximal finger joints. ${ }^{1}$ The disorder occurs at low prevalence worldwide but, due to an apparent founder effect, DTD is particularly prevalent in genetically isolated Finland, where it is the most common form of dwarfism.

We previously identified the gene mutated in DTD by positional cloning. ${ }^{2}$ The gene encodes a sulfate transporter and was named diastrophic dysplasia sulfate transporter or DTDST. It produces a ubiquitously expressed mRNA of approximately $8.4 \mathrm{~kb}$ based on Northern blotting analysis, of which we reported $2.8 \mathrm{~kb}$ of cDNA sequence including an open reading frame 
predicted to encode a protein of 739 amino acids. Several mutations in the reported sequence were found in DTD patients.

We subsequently showed that DTDST mutations also underlie two other chondrodysplasias: atelosteogenesis type II $(\mathrm{AOII})^{3}$ and achondrogenesis type IB (ACG1B), ${ }^{4}$ both of which are recessive and perinatally lethal. DTDST mutations thus give rise to a clinical spectrum, with compound heterozygosity and mutational overlap observed: several mutations have been detected in two or three of the conditions. ${ }^{3-7}$ The severity of the phenotype appears to correlate with residual DTDST function, although additional factors can also influence expression as illustrated by intrafamilial phenotypic variability. ${ }^{8,9}$

Despite extensive characterization of DTDST mutations, we had previously been unable to identify the major founder mutation accounting for the high prevalence of DTD in the Finnish population.

Haplotype analysis of four nearby genetic markers (Figure 1) showed that $95 \%$ of Finnish DTD chromosomes carried a rare haplotype present in only $4 \%$ of control chromosomes, suggesting the presence of a founder mutation carried on these chromosomes. Moreover, experiments on cultured skin fibroblasts from Finnish patients homozygous for the haplotype demonstrated that DTDST mRNA was undetectable on northern blots and that sulfate uptake was drastically impaired. However, direct sequencing of the then known portions of the DTDST gene failed to reveal a mutation in such patients.

Identification of the Finnish founder mutation is of interest both for clinical practice, as it will permit carrier testing in a population with $1-2 \%$ carrier frequency (T Pastinen, personal communication) and for population genetics. To identify the founder mutation, we sought to isolate the substantial untranslated regions of DTDST in order to screen them for mutations in Finnish patients.

\section{Materials and Methods}

\section{$5^{\prime}$ and $3^{\prime}$ UTR extension}

To extend the DTDST sequence to $5^{\prime}$ and $3^{\prime}$ direction, both screening of cDNA libraries and rapid amplification of cDNA ends (RACE) were used. A human thymus cDNA library in lambda gt11 (Clontech, Palo Alto, California, USA), containing both oligo(dT)-primed and random primed clones was screened using ${ }^{32} \mathrm{P}$-labeled PCR fragments from both ends of known DTDST sequence as probes, according to standard procedures. In addition, an oligo(dT)-primed human brain library was screened using the Genetrapper cDNA positive selection system, according to the manufacturer's instructions (Life Technologies, Garthesburg, Maryland, USA). Positive clones were then sequenced. RACE was performed using placental RNA as the template. First strand cDNA for $3^{\prime}$ RACE was prepared using an adapter-primer containing a universal amplification sequence (UAS) and dT17 (GGC CAC GCG TCG ACT AGT ACT TTT TTT TTT TTT TTTT). A gene-specific primer (TCC AAG AGA TGA AGC GGT GAA G) near the published 3' end along with the universal amplification primer (UAP) (CUA CUA CUA CUA GGC CAC GCG TCG ACT AGT AC) was used in the amplification of the cDNA. This reaction produced about a 500 product, which was gel-purified, PCR amplified with a nested gene-specific primer (CUA CUA CUA CUA GAA TGT TGC ACC TGC TCT AG) and the UAP, cloned into the pAMP10 vector, and sequenced. The 3 ' end was extended further using the same procedure. The initial amplification employed the gene-specific primer (CAT GAA TTG AGG AGG AAT TTG C) and the nested amplification employed the gene-specific primer (CUA CUA CUA CUA CAA GAA TGT CCA ACA CTG AAA G) and produced an overlapping product of about $3800 \mathrm{bp}$.

The 5' RACE was carried out in similar fashion except first strand cDNA was primed with gene-specific primer (GTC AGT ACT TGA TTC CCT TTG) and tailed with dCTP and terminal transferase (Life Technologies). A nested genespecific primer (CUA CUA CUA CUA GAT CCC AGA TGG ATA ACT GTC) and an anchor primer (AP) (CUA CUA CUA CUA GGC CAC GCG TCG ACT AGT ACG

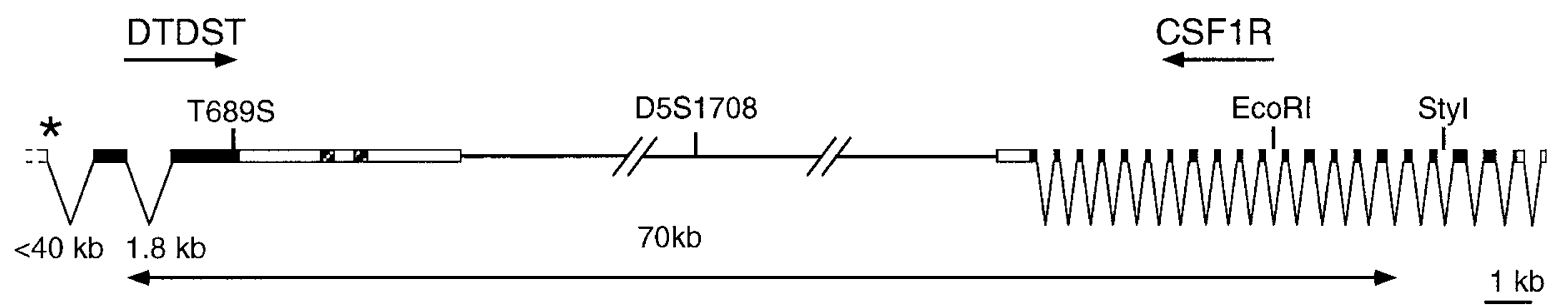

Figure 1 Structure of DTDST gene and polymorphisms used in haplotype analysis. The DTDST gene and the colony stimulating factor 1 receptor gene (CSF1R), which contains two of the polymorphisms used for haplotyping the DTD patients, are arranged tailto-tail approximately $70 \mathrm{~kb}$ from each other. Exons are depicted as boxes, with untranslated regions marked in white and translated regions in black. The two Alu repetitive elements in the 3' UTR of DTDST are hatched. The site of DTDST Fin is indicated with an asterisk. The locations of the four polymorphisms used for haplotype analysis (T689S at DTDST, D5S1708 and EcoRI at CSF1R and StyI at CSF1R) are shown. 
GGI IGG GII GGG IIG) were used. The PCR product was cloned into the pAMP1D vector and sequenced.

Genomic sequence preceding the 5' UTR and from exonintron junctions was determined by sequencing of genomic clones from the region.

\section{DTD Families}

A total of 84 Finnish DTD families was studied. Diagnoses were based on clinical and radiological findings typical for DTD, and confirmed by one of the authors (IK). High molecular weight DNA from venous blood, cultured lymphoblasts, or cultured fibroblasts was extracted according to standard protocols. ${ }^{10}$

\section{Sequencing of Patient DNA}

Untranslated regions of DTDST were amplified from genomic DNA in five fragments. The 5' UTR was amplified with the primer pair AGC ACT TCA AAA CTC TGG GC and GAC CCC TGA TCT GGG ATT CT. The 3' UTR was amplified with four sets of primer pairs: TTA TAC ATT TGG ACT GTG and CCC AAG TAG AGA GGA TGA AAT G; GTC CCA TCC ATC CAA ACA AG and TTT CTG CCT CCG CTG GTC; GTT ACA GCC AAA GGG TTG G and CCC CAT CCC TGT AAG TTA CTA A; and GTT TCA CTA GCA GGG AAG TTT C and AGC AGA CAA AAC AAA TGA AAC C. Hemi-nested PCR was then performed with a chimeric primer having an M13-sequencing primer site at its $5^{\prime}$ end. The resulting fragments were then sequenced by standard dideoxy sequencing using a dye-labeled primer complementary to the M13 primer site, and the resulting sequencing reaction was assayed on an ABI373 sequencer (Applied Biosystems, Foster City, California, USA).

\section{PCR Restriction Assay for DTDST $T_{\text {Fin }}$ Mutation}

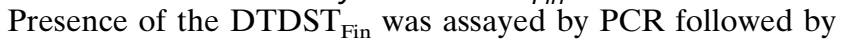
restriction digestion as follows: $100 \mathrm{ng}$ genomic DNA was amplified with primers CCG GCA GTA CCA CGT GAC and GAC CCC TGA TCT GGG ATT CT in a reaction volume of $30 \mu \mathrm{l}$, with 35 cycles of $\left(94^{\circ} \mathrm{C} 30 \mathrm{~s}, 55^{\circ} \mathrm{C} 30 \mathrm{~s}, 72^{\circ} \mathrm{C} 90 \mathrm{~s}\right)$ followed by $10 \mathrm{~min}$ extension at $72^{\circ} \mathrm{C}$. The resulting $381 \mathrm{bp}$ PCR products were digested with HphI (New England Biolabs, Beverly, Massachusetts, USA) according to the manufacturer's instructions. The fragments were fractionated in 3\% agarose gel, stained with ethidium bromide and visualized with UV light. The wild type allele is cut into two fragments of $269 \mathrm{bp}$ and $112 \mathrm{bp}$ in size while the mutant allele remains intact.

\section{Quantitation of DTDST mRNA by RT-PCR}

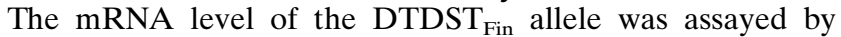
RT-PCR in two ways. Both approaches began with oligo dT-primed first-strand cDNA synthesis from total skin fibroblast RNA, using the Superscript II preamplification system (Life Technologies) according to the manufacturer's instructions.

The first approach involved simultaneous amplification of RT-PCR assays from DTDST (288 bp) and, as a control, the transferrin receptor $(T F R)$ gene $(1347 \mathrm{bp})$. Both assays spanned exon boundaries to ensure that only cDNA rather than residual genomic DNA was assayed. The ratio of DTDST:TFR products provided an estimate of the transcription level of the DTDST gene in different samples. The RT-PCR amplification was performed in a single $20 \mu \mathrm{l}$ reaction containing either $0.2 \mu \mathrm{l}$ or $2 \mu \mathrm{l}$ of $1: 10$ dilution of
cDNA template and 2 pmol of each primer (DTDST: TGC TCC TTC CTT AGG AAT GG and TGG CTT CAT AAT CTC TGC GA) (TFR: CCA CCA TCT CGG TCA TCA GGA TTG CCT and TTC TCA TGG AAG CTA TGG GTA TCA CAT). Cycling times were $94^{\circ} \mathrm{C} 1 \mathrm{~min}, 55^{\circ} \mathrm{C} 1.5 \mathrm{~min}$, and $72^{\circ} \mathrm{C} 2.5 \mathrm{~min}$ and the number of cycles was 20 (see Figure 3a).

The second approach involved a semi-quantitative mixing experiment, exploiting a polymorphism in the DTDST gene. Specifically, the general population has a T/S polymorphism at amino acid 689 (encoded by A/T polymorphism at nucleotide 2092 , which can be readily assayed because it creates an EcoNI restriction site). All chromosomes bearing DTDST Fin carry the S allele. cDNA was prepared from two samples: (i)

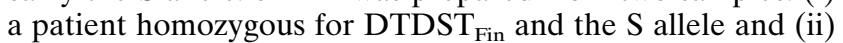
a normal individual homozygous for DTDST $_{w t}$ and the $\mathrm{T}$ allele. The concentration of the two cDNA samples was first adjusted so that they yielded equivalent levels of the $1.37 \mathrm{~kb}$ RT-PCR product from TFR. This was carried out by setting up a $50 \mu$ l PCR reaction from each sample, which was amplified for $15,20,25,30$, and 35 cycles, after which aliquots were removed. Band intensities were measured with Fluor-S MultiImager System (Bio-Rad, Hercules, California, USA) and the template concentrations adjusted accordingly. Then the two samples were mixed in the ratio $1: 2^{-i}$, for $i=1,2,3, \ldots$, 8 , and amplified with DTDST primers, TGG TTG GCA GCA CTG TAA CC and GAC TCA GAC CAT TGG GAA CAC, producing a $1.55 \mathrm{~kb}$ RT-PCR product that spans the second intron and contains the polymorphic site. The ratio of products derived from the two samples was assayed after digestion with EcoNI (which cleaves the S into 1411 and $134 \mathrm{bp}$ fragments, but does not cut the $\mathrm{T}$ allele) followed by electrophoresis in a $1 \%$ agarose gel and staining with ethidium bromide (see Figure $2 \mathrm{~b}$ ).

The mRNA level of the R279W allele was assayed by performing RT-PCR (of a segment spanning an intron, to distinguish RNA from genomic DNA) on a sample from an unaffected parent heterozygous for the R279W mutation and a normal allele. The resulting RT-PCR product was then subjected to DNA sequencing, to estimate the relative proportion of the two alleles represented in the mRNA. RT-PCR was carried out with DTDST primers TGC TCC TTC CTT AGG AAT GG and TGG CTT CAT AAT CTC TGC GA, with the latter primer biotinylated. The doublestranded, biotinylated PCR product was captured with streptavidin-coated magnetic beads (Dynal, Oslo, Norway) and processed according to the manufacturer's instructions to remove the non-biotinylated strand. The resulting singlestranded PCR product was sequenced with Sequenase polymerase (Sequenase, Pharmacia, Uppsala, Sweden) and $\alpha-\left[35^{\mathrm{S}}\right]-\mathrm{dATP}$.

\section{Results}

\section{DTDST Gene}

The previously determined part of the transcription unit of the DTDST gene was extended in both 5' and 3' directions by three approaches: isolation of clones from human thymus and brain cDNA libraries; RACE on placental cDNA; and analysis of ESTs from public databases. 

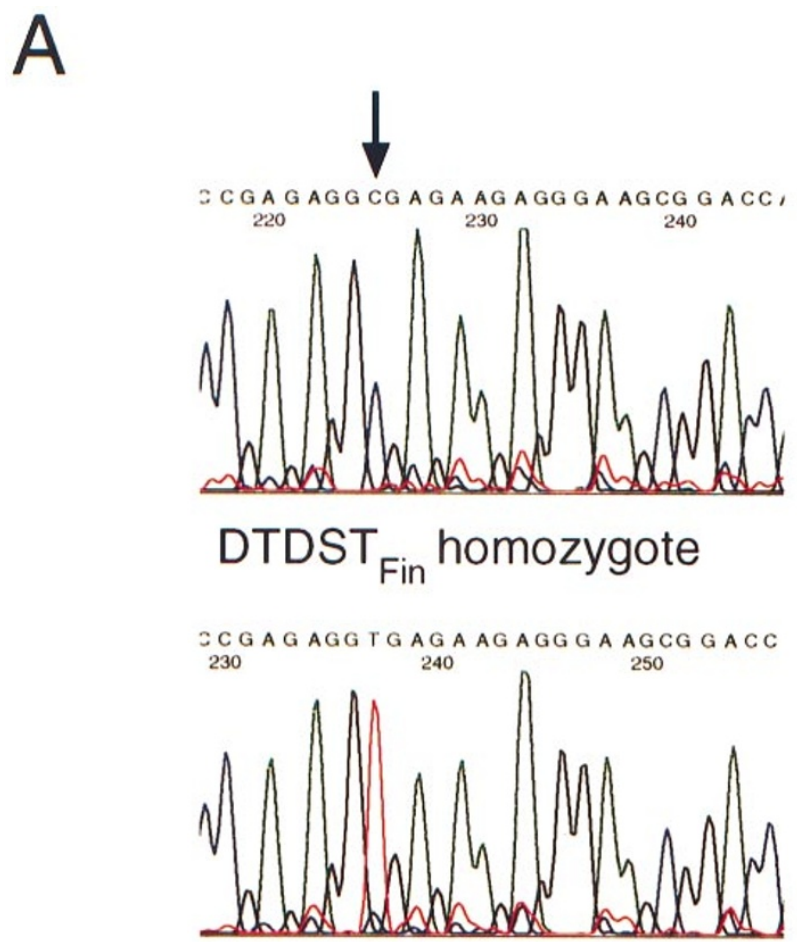

wt homozygote

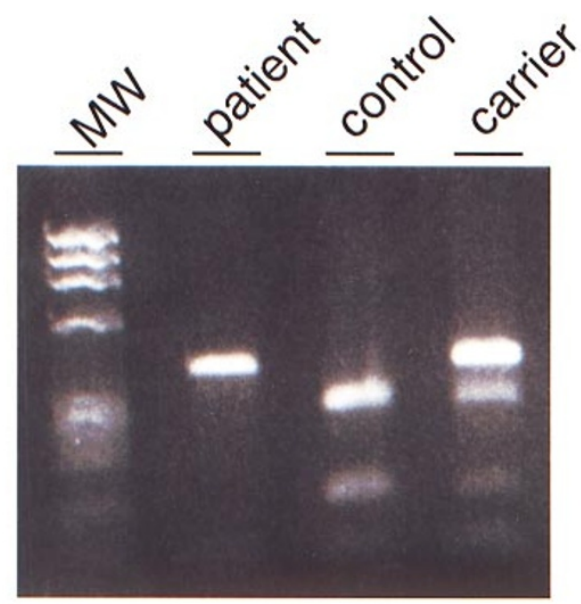

Figure 2 The sequence of and a restriction assay for the ${ }_{D T D S T} T_{\text {Fin }}$ mutation. A Splice donor site sequence of 5 ' noncoding exon containing the DTDST $T_{\text {Fin }}$ from a homozygous patient (first panel) and from a non-carrier sibling (second panel). The mutation is indicated with an arrow. B PCR-HphI restriction assay for DTDST $T_{F i n}$ mutation. MW: HaeIII digest of PhiX174, patient: DTDST Fin homozygote, control: DTDST $T_{w t}$ homozygote, and carrier: DTDST $T_{F i n} / D T D S T_{w t}$ heterozygote
The 3' UTR was found to extend for $4999 \mathrm{bp}$ of genomic DNA beyond the $3^{\prime}$ end of the previously published DTDST sequence, bringing the total length of the 3' UTR to $5584 \mathrm{bp}$. A polyadenylation signal starts at nucleotide 5562, and several cDNA clones were found containing corresponding polyA tracts $22 \mathrm{bp} 3^{\prime}$ of the polyadenylation signal. The $3^{\prime}$ UTR is devoid of introns but contains two Alu repeats arranged in head-to-head orientation with $479 \mathrm{bp}$ of non-repetitive sequence between them. The conclusion that the 3' UTR contains no introns was initially complicated by the fact that the two Alu repeats and the region between them appear readily to delete during cDNA preparation and cloning. Specifically, $4 \mathrm{cDNA}$ clones and 59ESTs were identified corresponding to the 3' UTR. These clones covered the great majority of the 3' UTR regions on either side of the Alu repeats. cDNA clones were found that crossed one of the repeats and contained some of the inter-repeat region. Cloned RACE products had the inter-Alu and parts of both Alu repeats deleted. The 'deleted' cDNA clones represent cloning artifacts rather than legitimate splicing, based on three lines of evidence. First, four such clones were sequenced, and each showed a slightly different deletion. Second, no consensus splice signals are present near the deletion boundaries. Third, Northern hybridization of a probe contained entirely within the inter-Alu region detects the $8.4 \mathrm{~kb}$ band expected from $D T D S T$ mRNA. We thus concluded that the interrepeat region is contained in the mature mRNA.

The 5' UTR was extended by $217 \mathrm{bp}$ in the $5^{\prime}$ direction, based on both identification of cDNA clones and 5' RACE analysis. This 5' UTR sequence is contained within an exon that is spliced to the third base of the previously reported DTDST cDNA sequence. The presence of an exon boundary had not previously been apparent from comparison with genomic DNA, because the first two bases of the previously reported cDNA sequence happen to match the preceding two bases of the genomic DNA. However, it is now clear that these two bases in the cDNA derive from an upstream exon. The 5' UTR is GC rich with approximately $70 \%$ GC content.

The additional 5' and 3' UTR extended the length of the DTDST transcription unit to $8049 \mathrm{bp}$. This falls somewhat short of the $8.4 \mathrm{~kb}$ length estimated from northern blot analysis, indicating that some additional sequences may still be missing at the $5^{\prime}$ end. However, the methods employed here were unable to extend the gene beyond the single 5' untranslated exon of $221 \mathrm{bp}$. 
Satoh and co-workers recently characterized the rat homologue of DTDST and found three small 5' untranslated exons of 209, 134 and $129 \mathrm{bp}^{11}$ These untranslated exons do not show significant sequence similarity to the observed $5^{\prime}$ untranslated exon in the human.

\section{Mutation Analysis of Patients}

To identify the Finnish founder mutation, the DTDST gene was sequenced in two Finnish patients homozygous for the common ancestral haplotype, as well as in a non-carrier sibling. The first two bases of intron 1 were found to contain a mutation changing the invariant GT of the 5' splice donor site to GC. The mutation abolishes an HphI restriction site, which provides a convenient assay for mutation screening (Figure 1, 2a, and $2 b)$.

We analyzed 84 Finnish DTD families, consisting of 102 DTD patients and their 121 available parents. The mutation was present on 152 of the 168 independent DTD chromosomes transmitted to patients but on none of the parents' non-DTD chromosomes, indicating that the transition is disease-causing rather than merely a polymorphism.

The designation of this mutation recommended by the nomenclature working group for human gene mutations $^{12}$ is c. $-26+2 \mathrm{~T}>\mathrm{C}$, but, for convenience, we will refer to this Finnish founder mutation as DTDST $_{\text {Fin. }}$ Of the 84 Finnish DTD families, patients carried two copies of DTDST $\mathrm{Din}_{\text {in }} 69$ families, one copy in 14 families, and no copy in one family. We also analyzed 40 non-Finnish DTD patients from the United States and Europe and found 14 of their 80 disease alleles $(18 \%)$ to be DTDST Fin $_{\text {. }}$.

Of the 16 Finnish DTD chromosomes that did not carry DTDST $_{\text {Fin }}$, seven were not expected to carry the founder mutation inasmuch as they shared a haplotype that differed completely from the rare ancestral Finnish haplotype. By sequencing of currently known portions of the DTDST gene, we were not able to identify the mutation carried on this haplotype, indicating that it is likely to lie in the remaining 5' UTR or in regulatory sequences.

The fact that the remaining nine chromosomes did not carry DTDST $_{\text {Fin }}$ came as a surprise, however, inasmuch as they did carry the rare ancestral Finnish haplotype. Eight of the nine chromosomes were predicted to carry an R279W amino acid substitution, resulting from a CGG-> TGG codon range. The ninth chromosome was instead found to have a 3 bp deletion (1045-1047delGTT) resulting in an amino acid deletion
(V340del) in the predicted seventh transmembrane domain of the DTDST protein. Both these mutations have been previously identified in DTDST-related disorders R279W in DTD and AOII and V340del in ACG1B. ${ }^{3-7,13}$ In the one family carrying no copies of DTDST $_{\text {Fin }}$, the patient was a compound heterozygote for R279W/V340del.

Our original analysis based on haplotypes thus overestimated the proportion of chromosomes carrying the ancestral mutation by about $5 \% .{ }^{14}$ The proportion of ancestral mutations was used to estimate the distance to the mutation based on a Luria-Delbrück analysis. Fortunately, the slight overestimate had no significant impact on the estimate.

\section{$D T D S T_{\text {Fin }}$ Decreases mRNA Levels}

In our initial report on positional cloning of DTDST we noted that two patients presumed to be homozygous for the Finnish founder mutation (by virtue of being homozygous for the ancestral haplotype) showed a drastic reduction in the expression level of $D T D S T .^{2}$ Specifically, DTDST mRNA was undetectable on northern blots prepared from skin fibroblasts.

With the subsequent recognition that DTD, AOII and ACG1B form an allelic series with ACG1B having the most severe phenotype, it was predicted that the Finnish founder mutation could not completely abolish expression. We therefore used a more sensitive assay of mRNA expression, involving semi-quantitative reversetranscription polymerase chain reaction (RT-PCR) with an internal control gene (see Materials and Methods). This assay revealed that patients homozygous for DTDST $_{\text {Fin }}$ have a low but detectable level of correctly spliced mRNA, estimated to be approximately $5 \%$ of the wild-type allele (Figure $2 \mathrm{a}$ and $2 \mathrm{~b}$ ). Correct splicing was confirmed by sequencing across the affected splice junction (data not shown).

In contrast to the DTDST Fin allele, the R279W allele showed wild-type mRNA levels, as assayed by RT-PCR analysis of a sample from an individual heterozygous for R279W and a normal allele (see Materials and Methods) (Figure 3c).

\section{Discussion}

We have identified more than $5 \mathrm{~kb}$ of additional sequence from the 5' and 3' UTRs of the DTDST gene. the 3' UTR appears to be complete, but the 5' UTR may still be incomplete. These regions were then resequenced in Finnish DTD patients, in order to identify the Finnish founder mutation. 
A

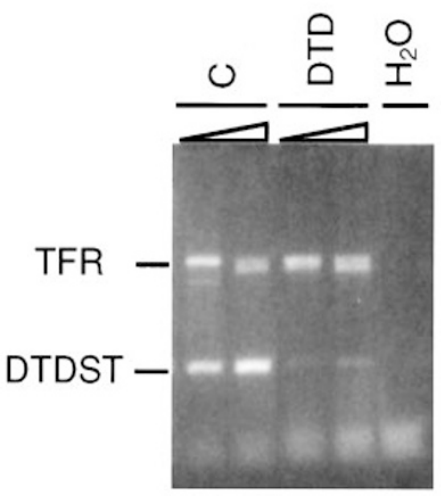

B

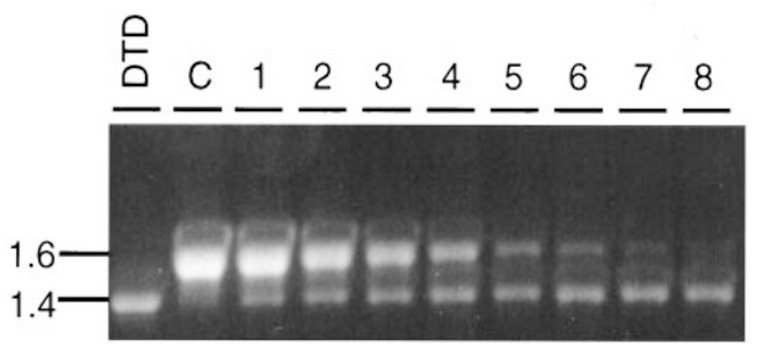

C

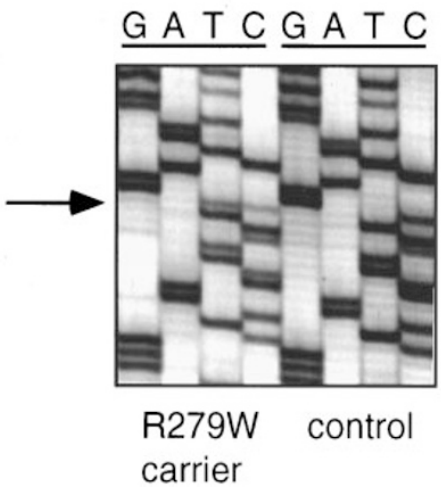

Figure 3 The $m R N A$ expression is reduced by the DTDST $T_{F i n}$ allele but not by the $R 279 \mathrm{~W}$ allele. A RT-PCR analysis of fibroblasts from an unaffected control $(C)$ and a diastrophic dysplasia (DTD) patient homozygous for the DTDST $T_{\text {Fin }}$ allele, involving simultaneous amplification of a $288 \mathrm{bp}$ PCR product across an exon boundary of DTDST and a $1347 \mathrm{bp}$ product across an exon boundary of transferrin receptor gene (TFR) as an internal control. DTDST expression is drastically decreased, but just detectable. Each sample was amplified using two different template concentrations (See text in Materials and Methods). Genomic DNA (data not shown) and water controls were included in the experiment. B RT-PCR for quantitation of expression of DTDST Fin allele. Lanes marked DTD and C contain RT-PCR products from, respectively, DTDST $T_{\text {Fin }}$ homozygote and wild-type homozygote. Lanes $i(i=1,2, \ldots, 8)$ contain a mixture in the ratio $1: 2^{-i}$ of a constant amount of cDNA from a DTDST $T_{\text {Fin }}$ homozygote with decreasing amounts of $c D N A$ from a wild-type homozygote. After PCR, the resulting product was digested with EcoNI, which cuts the $D_{T D S T}$ Fin allele, but leaves the wild-type allele intact. The intensities of mutant and wild-type bands are approximately equal in lanes 4 and 5, indicating that the DTDST Fin allele is reduced by $2^{-4}$ to $2^{-5}$-fold - that is, to 6-3\% of wildtype levels. C Sequence analysis of RT-PCR product from a DTD carrier heterozygous for the $\mathrm{R} 279 \mathrm{~W}$ allele and a wild-type allele. The site of the mutation is indicated with an arrow. The R279W mutant and the wild-type sequence is of roughly equal intensity in the carrier, indicating approximately equal expression levels of the two alleles.

The Finnish founder mutation was previously known to cause severely reduced expression level, but was not expected to abolish gene function completely (inasmuch as the null phenotype of DTDST is the perinatal lethal disorder ACG1B, rather than the viable disease DTD). Consistent with this expectation, 90\% of Finnish DTD chromosomes were found to have a mutation (GT-> GC) affecting the first two bases of the 5' splice donor of intron 1 .

Possible consequences of splice site mutations include exon skipping, activation of cryptic splice sites, creation of pseudo-exons within introns, and intron retention. ${ }^{15-17}$ Mutant mRNA levels are often low due to accelerated degradation, reduced synthesis, or disturbed transport to cytosol. No aberrant splice forms of DTDST were detected on a Northern blot, but a low level (about 5\%) of correctly spliced mRNA was detected by RT-PCR.

The presence of a GT-> GC mutation is indeed consistent with low, but non-zero expression. In vitro studies on the effects of point mutations on the invariant GT at the 5' splice donor site found that a GT-> GC mutation is the only single base change that resulted in correct splicing, albeit at reduced efficiency. ${ }^{18}$ Moreover, among thousands of splice sites studied, there is a small minority in which the GT is replaced by $\mathrm{GC}^{19}$

A surprising finding was that nine DTD chromosomes previously predicted to carry the Finnish founder mutation (by virtue of carrying a rare haplotype present on only $4 \%$ of control chromosomes) carried

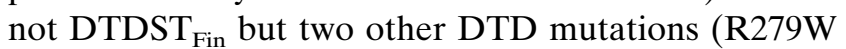
in eight cases and V340del in one case).

The fact that DTDST $_{\text {Fin }}$ happened to occur on any given rare haplotype is easily ascribed to chance, but the fact that the second most common DTD mutation in Finland (R279W) and a third mutation (V340del) 
also occurred on chromosomes bearing the same haplotype at nearby markers seems puzzling. We can only speculate about possible explanations.

One possibility is that the three DTD mutations arose in a population in which this 'rare' haplotype was in fact common and conferred a heterozygote advantage in this population, resulting in an excess of DTD mutations on this haplotype which were then admixed with a larger population in which the haplotype was rare. Although such a scenario could account for the presence of three mutations on this haplotype, there is no evidence to support the existence of such an ancestral population or of a heterozygote advantage conferred by DTD chromosomes. A second possibility is that the chromosomes with the rare haplotype are more prone to mutation, although again there is no evidence to support such a hypothesis. Examples are known of chromosomes prone to triplet-repeat expansion in the germline ${ }^{20}$ or to APC mutations in somatic cells, ${ }^{21}$ but neither provides a close precedent for the case at hand. Finally, a third possibility is that the presence of multiple mutations on the rare haplotype is simply a matter of random chance.

The most immediate practical consequence of our work is that it permits carrier and prenatal screening in the Finnish population. Until now, screening has been possible only by linkage analysis in families with an affected individual. A test for DTDST $_{\text {Fin }}$ and R279W would identify $95 \%$ of all DTD-causing chromosomes in Finland.

\section{Acknowledgements}

We wish to thank patients and their families who participated in this study. This work was supported in part by grants from the Academy of Finland, The Sigrid Juselius Foundation, the Ulla Hjelt foundation to JH and the US National Institutes of Health (AR41970 to AC, HG00368 to ML, and HG0098 to ESL).

\section{References}

1 Walker BA, Scott CI, Hall JG, Murdoch JL, McKusick VA: Diastrophic dwarfism. Medicine 1972; 51: 41-59.

2 Hästbacka J, de la Chapelle A, Mahtani MM et al: The diastrophic dysplasia gene encodes a novel sulfate transporter: positional cloning by fine-structure linkage disequilibrium mapping. Cell 1994; 78: 1073-1087.

3 Hästbacka J, Superti-Furga A, Wilcox WR, Rimoin DL, Cohn DH, Lander ES: Atelosteogenesis type II is caused by mutations in the diastrophic dysplasia sulfate transporter gene (DTDST): Evidence for a phenotypic series involving three chondrodysplasias. Am J Hum Genet 1996; 58: $255-262$.
4 Superti-Furga A, Hästbacka J, Wilcox W et al: Achondrogenesis type IB is caused by mutations in the diastrophic dysplasia sulfate transporter gene. Nat Genet 1996; 12: 100-102.

5 Superti-Furga A, Rossi A, Steinmann B, Gitzelmann R: A chondrodysplasia family produced by muations in the diastrophic dysplasia sulfate transporter gene: genotype/ phenotype correlations. Am J Med Genet 1996; 63: 144-147.

6 Rossi A, van der Harten HJ, Beemer FA et al: Phenotypic and genotypic overlap between atelosteogenesis type 2 and diastrophic dysplasia. Hum Genet 1996; 98: 657-661.

7 Rossi A, Bonaventure J, Delezoide AL, Superti-Furga A, Cetta G: Undersulfation of cartilage proteoglycans ex vivo and increased contribution of amino acid sulfur to sulfation in vivo in McAlister dysplasia/atelosteogenesis type 2. Eur J Biochem 1997; 248: 741-747.

8 Horton WA, Rimoin DL, Lachman RS et al: The phenotypic variability in diastrophic dysplasia. $J$ Pediatr 1978; 93: 609-613.

9 Hall B: Diastrophic dysplasia: extreme variability within a sibship. Am J Med Genet 1996; 63: 28-33.

10 Sambrook J, Fritsch EF, Maniatis T: Molecular Cloning, a Laboratory Manual. Cold Spring Harbor Laboratory Press: Cold Spring Harbor, New York, 1989.

11 Satoh H, Susaki M, Shukunami C, Iyama K, Negoro T, Hiraki Y: Functional analysis of diastrophic dysplasia sulfate transporter. $J$ Biol Chem 1998; 273: 12307-12315.

12 Antonorakis S, and the Nomenclature working group: Recommendations for a nomenclature system for human gene mutations. Hum Mutat 1998; 11: 1-3.

13 Cai G, Nakayama M, Hiraki Y, Ozono K: Mutational analysis of the DTDST gene in a fetus with achondrogenesis type 1B. Am J Med Genet 1998; 78: 58-60.

14 Hästbacka J, de la Chapelle A, Kaitila I, Sistonen P, Weaver A, Lander ES: Linkage disequilibrium mapping in isolated founder populations: diastrophic dysplasia in Finland. Nat Genet 1992; 2: 343.

15 Krawczak M, Reiss J, Cooper DN: The mutational spectrum of single base-pair substitutions in mRNA splice junctions of human genes: causes and consequences. Hum Genet 1992; 90: 41-54.

16 Berget SM: Exon recognition in vertebrate splicing. J Biol Chem 1995; 270: 2411-2414.

17 Maquat LE: Defects in RNA splicing and the consequence of shortened translational reading frames. Am J Hum Genet 1996; 59: 279-286.

18 Aebi M, Horing $\mathrm{H}$, Weissmann C: 5' Cleavage site in eukaryotic pre-mRNA splicing is determined by the overall 5' splice region, not by the conserved 5' GU. Cell 1987; 50: 237-246.

19 Jackson IJ: A reappraisal of non-consensus mRNA splice sites. Nucleic Acids Res 1991; 19: 3795-3798.

20 Cossee M, Schmitt M, Campuzano V et al: Evolution of the Friedreich's ataxia trinucleotide repeat expansion: founder effect and premutations. PNAS 1997; 94: 7452-7457.

21 Laken SJ, Petersen GM, Gruber SB et al: Familial colorectal cancer in Ashkenazim due to a hypermutable tract in APC. Nat Genet 1997; 17: 79-83. 